\title{
Cardiovascular surgery risk prediction from the patient's perspective
}

\author{
Hiroaki Miyata, PhD, Noboru Motomura, MD, PhD, Ryohei Yozu, MD, PhD, Shunei Kyo, MD, PhD, and \\ Shinichi Takamoto, MD, PhD, for the Japan Cardiovascular Surgery Database
}

\begin{abstract}
Objective: Previous studies have developed cardiovascular surgery outcome prediction models using only patient risk factors, but surgery outcomes from the patient's perspective seem to differ between hospitals. We have developed outcome prediction models that incorporate preoperative patient risks, as well as hospital processes and structure.
\end{abstract}

\begin{abstract}
Methods: Data were collected from the Japan Cardiovascular Database for patients scheduled for cardiovascular surgery between January 2005 and December 2007. We analyzed 33,821 procedures in 102 hospitals. Logistic regression was used to generate risk models, which were then validated through split-sample validation.

Results: Odds ratios, 95\% confidence intervals, and $P$ values for structures and processes in the mortality prediction model were as follows: "hospital annual adult cardiac surgery volume (continuous; every 1 procedure increase per year)" (odds ratio, 0.998; confidence interval, 0.997-0.999; $P<.001$ ); "recommended staffing and equipment" (odds ratio, 0.75 ; confidence interval, $0.64-0.87 ; P<.001$ ); "daily conferences with cardiologists" (odds ratio, 0.79; confidence interval, $0.60-1.02 ; P=.073$ ); "intensivists involved in postsurgical management" (odds ratio, 0.89; confidence interval, 0.77-1.02; $P=.90$ ); "public hospitals" (odds ratio, 0.80; confidence interval, $0.70-0.93 ; P=.003$ ); "surgeons lacking miscellaneous duties" (odds ratio, 0.80; confidence interval, $0.70-0.93 ; P=.003$ ); and "surgeons who work no more than 32 hours per week" (odds ratio, 0.55 ; confidence interval, $0.32-0.95 ; P=.032$ ). The mortality prediction model had a C-index of 0.85 and a Hosmer-Lemeshow $P$ value of .79 .
\end{abstract}

Conclusions: Our models yielded good discrimination and calibration, so they may prove useful for hospital selection based on individual patient risks and circumstances. Improved surgeon work environments were also shown to be important for both surgeons and patients. (J Thorac Cardiovasc Surg 2011;142:e71-6)

Patient outcome evaluation is gaining acceptance as a first step in the assessment and improvement of healthcare quality. Prior studies identified predictors of mortality and morbidity, ${ }^{1-6}$ but many of these models were developed using only patient risk factors. Given that most of these models were created for healthcare quality benchmarking, their predicted outcomes should not rely on the particular hospital in which the procedure was performed. However, from the patient's perspective, predicted outcomes of cardiovascular surgery seem to differ between hospitals, a perception that has been proven accurate.

Many studies have suggested that patients undergoing cardiovascular surgery at high-volume hospitals have lower risks of operative mortality compared with patients at lower-volume centers. ${ }^{7-11}$ Hospital volume is used as a surrogate measure for numerous processes of care that directly influence patient outcomes because individual processes have not been well characterized and are

From the Japan Cardiovascular Surgery Database, Tokyo, Japan.

Disclosures: Authors have nothing to disclose with regard to commercial support.

Received for publication Sept 22, 2009; revisions received Dec 6, 2010; accepted for publication Jan 10, 2011; available ahead of print Feb 21, 2011.

Address for reprints: Hiroaki Miyata, PhD, 7-3-1 Hongo, Bunkyo-ku, Tokyo 113-

8655, Japan (E-mail: hiroaki.miyata@gmail.com).

$0022-5223 / \$ 36.00$

Copyright (c) 2011 by The American Association for Thoracic Surgery

doi:10.1016/j.jtcvs.2011.01.018 difficult to measure. For some procedures, higher volume, particularly at the surgeon level, may translate directly to better clinical judgment and technical proficiency in the operating room. Such processes may be hard to transfer to lower-volume providers. On the other hand, high-volume and low-volume centers may also differ with regard to other processes of preoperative and postoperative care, which could be adopted by lower-volume centers. ${ }^{12}$

To better understand the volume-outcome relationship in cardiovascular surgery, we focused on practices that involve cardiovascular procedures, including hospital processes and structure, which embody potential risks of perioperative mortality. These models may be useful not only for cardiologists considering treatment indication and referral but also for patients, because they may assist patients in site selection.

\section{MATERIALS AND METHODS Study Population}

The Japan Adult Cardiovascular Surgery Database (JACVSD) was initiated in 2000 to estimate surgical outcomes after cardiovascular procedures in many centers throughout Japan. The JACVSD adult cardiovascular division currently captures clinical information from approximately half of all Japanese hospitals performing cardiovascular surgery. The data collection form has 255 variables (definitions are available online at http://www. jacvsd.umin.jp), and these are almost identical to those in the Society of Thoracic Surgeons National Database (definitions are available online at 


$$
\begin{aligned}
& \text { Abbreviations and Acronyms } \\
& \begin{aligned}
\mathrm{CI} \quad= & \text { confidence interval } \\
\mathrm{JACVSD}= & \text { Japan Adult Cardiovascular Surgery } \\
& \text { Database } \\
\mathrm{OR} \quad= & \text { odds ratio }
\end{aligned}
\end{aligned}
$$

http://sts.org). The JACVSD has developed software for the web-based data collection system through which the data manager of each participating hospital submits their data electronically to the central office. Although participation in the JACVSD is voluntary, data completeness is a high priority. Accuracy of submitted data was maintained by a data audit that was achieved by monthly visits by administrative office members to the participating hospital to check data against clinical records. Validity of data was further confirmed by an independent comparison of the volume of cardiac surgery at a particular hospital entered in the JACVSD with that reported in the annual survey by the Japanese Association for Thoracic Surgery. ${ }^{13}$

We examined all cardiovascular surgery procedures between January 1 , 2005, and December 31, 2007. JACVSD records that had been obtained without patients' informed consent were excluded from this analysis. Records with missing age (or out of range), sex, or 30-day status (see "End Points") were also excluded. With the exception of body surface area, sex-specific median values were entered for all missing or out-of-range values. Although participation in the JACVSD is voluntary, data completeness is high, and less than $3 \%$ entries lack overall preoperative risk factors used in this study. After this data cleaning, the population for this risk model analysis consisted of 33,821 cardiovascular procedures from 102 participating sites throughout Japan. On the basis of interviews with cardiovascular surgeons and former studies (references), we developed items regarding structures (resources and administration) and processes (culture and professional cooperation) that may affect cardiovascular surgery outcomes.

\section{End Points}

The primary outcome measure of the JACVSD was 30-day operative mortality, which was defined exactly the same as the 30-day operative mortality in the Society of Thoracic Surgeons National Database. This includes any patient who died during the index hospitalization, regardless of the length of hospital stay, and any patient who died within 30 days of the operation after being discharged from the hospital. By using the definition from a previous study, ${ }^{14,15}$ major morbidity was defined as any of the following 5 postoperative in-hospital complications: stroke, reoperation for any reason, need for mechanical ventilation for more than 24 hours after surgery, renal failure, or deep sternal wound infection.

\section{Statistical Analysis}

Data $(\mathrm{n}=33,821)$ were randomly divided into 2 subsets for model development $(27,191$ records, $80 \%)$ and validation tests (6630 records, $20 \%$ ). The statistical model was multiple logistic regression; variables entered in the model were selected from all variables shown in Tables 1 and 2 using bivariate tests. Chi-square tests analyzed categoric covariates, and unpaired $t$ tests or Wilcoxon rank-sum tests were used for continuous covariates. All variables significant at the $P<.2$ level were entered into the model. This model considered volume a continuous variable. A multivariate stepwise logistic regression analysis was then performed for each outcome. Stability of the model was checked every time a variable was eliminated. When all statistically nonsignificant variables had been eliminated from the model, "goodness-of-fit" was evaluated and the area under the receiver operating characteristic curve was used to assess how well the model could discriminate between patients who lived and patients who had died. ${ }^{16}$ Model calibration (the degree to which observed outcomes were similar to the predicted outcomes across patient groups) was evaluated by comparing the observed with the predicted mean values within each of 10 equal-sized subgroups arranged in order of increasing patient risk. To evaluate model calibration, the Hosmer-Lemeshow test was applied.

\section{RESULTS \\ Patient Characteristics}

Patient characteristics and outcomes of each procedure are shown in Table 1. The 33,821 procedures consisted of the following: isolated CABG surgery $(n=10,972)$, valve surgery $(\mathrm{n}=10,935)$, thoracic aortic surgery $(\mathrm{n}=7559)$, and other procedures $(n=4355)$. Patient median age was 68.9 years (range 60.0-75.2 years), and the percentage of male patients per procedure was as follows: CABG surgery $(77.2 \%)$, valve surgery $(55.1 \%)$, thoracic aortic surgery $(64.5 \%)$, and other procedures $(57.3 \%)$. As shown in Table 3, 30-day operative mortality rates were $2.2 \%$ for isolated CABG surgery, $3.7 \%$ for valve surgery, $8.4 \%$ for thoracic aortic surgery, and $7.1 \%$ for other procedures. Composite rates for mortality or major morbidity were $14.2 \%$ for isolated CABG surgery, $15.5 \%$ for valve surgery, $31.7 \%$ for thoracic aortic surgery, and $7.1 \%$ for other procedures.

\section{Participant Hospital Characteristics}

The characteristics of participant hospitals are shown in Table 2. Of the 102 hospitals, $53.9 \%$ were public hospitals (managed by a local public organization), $45.1 \%$ were university hospitals (affiliated with a university), and 89.2\% were general hospitals (had various diagnosis and treatment departments, including at least an internal medicine department and a surgery department). Sixty-one $(63.7 \%)$ of the participating hospitals met staffing and equipment criteria recommended by previous research in Japan of having more than 4 cardiovascular surgeons, more than 2 expert cardiovascular surgeons, more than 4 cardiologists, more than 2 anesthesiologists, more than 2 clinical engineers, an intensive-care unit facility, a hemodialysis unit, and more than 2 heart-lung machines. Most participant hospitals $(97.1 \%)$ held more than 1 conference per week with cardiovascular surgeons.

\section{Model Results and Performance}

Two models were developed, and the final logistic model is presented in Table 4. Odds ratios (ORs), confidence intervals (CIs), and $P$ values for structures and processes in the mortality prediction model were as follows: "hospital annual adult cardiac surgery volume (continuous: every 1 procedure increase per year)" (OR, 0.998; CI, 0.997-0.999; $P<.001)$; "recommended staffing and equipment" (OR, 0.75 ; CI, 0.64-0.87; $P<.001)$; "daily conferences with cardiologists" (OR, 0.79; CI, 0.60-1.02; $P=.073$ ); "intensivists involved in postsurgical management" (OR, 0.89; CI, 0.77-1.02; $P=.90$ ); "public hospitals" (OR, 0.80; CI, 
TABLE 1. Characteristics of patients $(N=33,821)$

\begin{tabular}{|c|c|c|c|c|c|c|}
\hline & All patients $(\mathrm{n}=\mathbf{3 3 , 8 2 1})$ & \multicolumn{2}{|c|}{ Test data set $(\mathbf{N}=\mathbf{2 7 , 1 9 1 )}$} & & \multicolumn{2}{|c|}{ Validation data set $(\mathbf{N}=6630)$} \\
\hline Age $(y)$, median $(25-75)$ & $.0-75.2)$ & & $50.0-75.2)$ & & $69.0(59$ & \\
\hline BSA $\left(\mathrm{kg} / \mathrm{m}^{2}\right)$ median $(25-75)$ & $47-1.73)$ & & (.47-1.73) & & $1.60(1.4$ & \\
\hline & $\mathbf{n}$ & $\%$ & $\mathbf{n}$ & $\%$ & $\mathbf{n}$ & $\%$ \\
\hline Gender (male) & 21,909 & 64.8 & 17,575 & 64.6 & 4334 & 65.4 \\
\hline BMI $31-35 \mathrm{~kg} / \mathrm{m}^{2}$ & 923 & 2.7 & 759 & 2.7 & 163 & 2.7 \\
\hline $\mathrm{BMI}>35 \mathrm{~kg} / \mathrm{m}^{2}$ & 142 & 0.4 & 125 & 0.5 & 17 & 0.3 \\
\hline Smoking & 13,886 & 41.1 & 11,134 & 40.9 & 2752 & 41.5 \\
\hline Diabetes & 8778 & 26.0 & 7060 & 26.0 & 1718 & 25.9 \\
\hline Renal failure & 3807 & 11.3 & 3080 & 11.3 & 727 & 11.0 \\
\hline Renal failure requiring dialysis & 1682 & 5.0 & 1370 & 5.0 & 312 & 4.7 \\
\hline Cerebrovascular disease (recent) & 340 & 1.0 & 268 & 1.0 & 72 & 1.1 \\
\hline Infectious endocarditis & 705 & 2.1 & 549 & 2.0 & 156 & 2.4 \\
\hline COPD (moderate, severe) & 671 & 2.0 & 551 & 2.0 & 120 & 1.8 \\
\hline Extracardiac arteriopathy (peripheral) & 3398 & 10.0 & 2750 & 10.1 & 648 & 9.8 \\
\hline Extracardiac arteriopathy (thoracic) & 5992 & 17.7 & 4842 & 17.8 & 1150 & 17.3 \\
\hline Neurologic disorder & 1302 & 3.8 & 1055 & 3.9 & 247 & 3.7 \\
\hline Congestive heart failure & 8193 & 24.2 & 6589 & 24.2 & 1604 & 24.2 \\
\hline Cardiogenic shock & 1579 & 4.7 & 1269 & 4.7 & 310 & 4.7 \\
\hline NYHA IV & 2951 & 8.7 & 2362 & 8.7 & 589 & 8.9 \\
\hline Preoperative inotropic agents & 1676 & 5.0 & 1342 & 4.9 & 334 & 5.0 \\
\hline Triple vessel disease & 9120 & 27.0 & 7343 & 27.0 & 1777 & 26.8 \\
\hline Poor LV function & 1745 & 5.2 & 1397 & 5.1 & 348 & 5.2 \\
\hline Tricuspid stenosis & 85 & 0.3 & 70 & 0.3 & 15 & 0.2 \\
\hline Reoperation & 2126 & 6.3 & 1705 & 6.3 & 421 & 6.3 \\
\hline Status (urgent) & 2772 & 8.2 & 2213 & 8.1 & 559 & 8.4 \\
\hline Status (emergency) & 3480 & 10.3 & 2762 & 10.2 & 718 & 10.8 \\
\hline Surgery type "isolated CABG" (reference) & 10,972 & 32.4 & 8804 & 32.4 & 2168 & 32.7 \\
\hline CABG combined surgery & 3427 & 10.1 & 2783 & 10.0 & 699 & 10.5 \\
\hline Surgery type "valve" & 10,935 & 32.3 & 8839 & 32.5 & 2096 & 31.6 \\
\hline Aortic valve plus mitral valve & 1432 & 4.2 & 1138 & 4.2 & 294 & 4.4 \\
\hline Surgery type "thoracic aorta" & 7559 & 22.4 & 6089 & 22.4 & 1470 & 22.2 \\
\hline Thoracic aortic surgery indication (rupture) & 662 & 2.0 & 524 & 1.9 & 138 & 2.1 \\
\hline Range of replacement (root) & 922 & 2.7 & 729 & 2.7 & 193 & 2.9 \\
\hline Range of replacement (arch) & 3061 & 9.1 & 2470 & 9.1 & 591 & 8.9 \\
\hline Range of replacement (distal) & 1288 & 3.8 & 1042 & 3.8 & 246 & 3.7 \\
\hline Range of replacement (descending) & 1361 & 4.0 & 1114 & 4.1 & 247 & 3.7 \\
\hline Range of replacement (thoracoabdominal) & 553 & 1.6 & 451 & 1.7 & 102 & 1.5 \\
\hline Surgery type "other procedures" & 4355 & 12.9 & 3459 & 12.7 & 896 & 13.5 \\
\hline Resection/myoplasty & 157 & 0.5 & 119 & 0.4 & 38 & 0.6 \\
\hline Septal perforation/rupture & 314 & 0.9 & 255 & 0.9 & 59 & 0.9 \\
\hline
\end{tabular}

$B S A$, Body surface area; $B M I$, body mass index; $C O P D$, chronic obstructive pulmonary disease; $N Y H A$, New York Heart Association; $L V$, left ventricle; $C A B G$, coronary artery bypass grafting.

0.70-0.93; $P=.003)$; "surgeons who lack miscellaneous duties" (OR, 0.80; CI, 0.70-0.93; $P=.003)$; and "surgeons who do not perform more than 32 hours of continuous labor" (OR, 0.55; CI, 0.32-0.95; $P=.032$ ).

Model performance was evaluated using the C-index (area under the receiver operating characteristic curve) as a measure of model discrimination and the HosmerLemeshow test as a measure of "goodness-of-fit." The C-index was 0.85 for the mortality model and 0.74 for the composite mortality or morbidity model; the HosmerLemeshow test $P$ value was .79 for the mortality model and 0.49 for the composite mortality or morbidity model. Details of model performance metrics are shown in Table 5.

\section{DISCUSSION}

In this study, we developed a cardiovascular surgery risk prediction model from the patient perspective. Results of the C-index and Hosmer-Lemeshow test indicate good discrimination and calibration of the models. Patients may use these models to select a hospital according to their individual risks and circumstances. Choosing the optimal hospital also may be based on factors such as patient travel times and 
TABLE 2. Characteristics of hospital $(\mathbf{N}=102)$

\begin{tabular}{|c|c|c|}
\hline \multirow{2}{*}{$\begin{array}{c}\text { Hospital annual adult cardiac surgery } \\
\text { volume, median }(25-75)\end{array}$} & \multicolumn{2}{|c|}{$115.3(70.8-168.1)$} \\
\hline & $\mathbf{n}$ & $\%$ \\
\hline Public hospital & 55 & 53.9 \\
\hline University hospital & 46 & 45.1 \\
\hline General hospital & 91 & 89.2 \\
\hline Recommended staffing and equipment* & 65 & 63.7 \\
\hline Autologous blood transfusion system & 88 & 86.3 \\
\hline Have an infection control team & 90 & 88.2 \\
\hline $\begin{array}{l}\text { Conference with cardiovascular surgeons } \\
\text { (every day) }\end{array}$ & 49 & 48.0 \\
\hline $\begin{array}{l}\text { Conference with cardiovascular surgeons } \\
\qquad(\geq 1 / \mathrm{wk})\end{array}$ & 99 & 97.1 \\
\hline Conference with cardiologists (every day) & 7 & 6.9 \\
\hline Conference with cardiologists $(\geq 1 /$ wk) & 74 & 72.5 \\
\hline Conference with nurse ( $\geq 1 /$ wk) & 47 & 46.1 \\
\hline Conference with anesthesiologist $(\geq 1 /$ wk) & 40 & 39.2 \\
\hline $\begin{array}{l}\text { Intensivists involved in postsurgical } \\
\text { management }\end{array}$ & 54 & 52.9 \\
\hline $\begin{array}{l}\text { Have some protocols regarding drug } \\
\text { selection }\end{array}$ & 37 & 36.3 \\
\hline $\begin{array}{l}\text { Have some protocols regarding drug dilution } \\
\text { method }\end{array}$ & 70 & 68.6 \\
\hline $\begin{array}{l}\text { Have some protocols regarding patients' } \\
\text { rehabilitation }\end{array}$ & 49 & 48.0 \\
\hline Routinely check head and aortic wall CT scan & 78 & 76.5 \\
\hline $\begin{array}{l}\text { Preoperative beta-blocker use for CABG } \\
\text { surgery (first choice) }\end{array}$ & 5 & 4.9 \\
\hline $\begin{array}{l}\text { Discharge beta-blocker use for CABG } \\
\text { surgery (first choice) }\end{array}$ & 12 & 11.8 \\
\hline $\begin{array}{l}\text { Discharge antiplatelet use for CABG surgery } \\
\text { (first choice) }\end{array}$ & 92 & 90.2 \\
\hline $\begin{array}{l}\text { Discharge anti-lipid use for CABG surgery } \\
\text { (first choice) }\end{array}$ & 15 & 14.7 \\
\hline ITA use for CABG surgery (first choice) & 99 & 97.1 \\
\hline $\begin{array}{l}\text { Off-pump procedure for CABG surgery } \\
\text { (first choice) }\end{array}$ & 49 & 48.0 \\
\hline Surgeons do not have miscellaneous duties & 59 & 57.8 \\
\hline Surgeons do not have $>32-\mathrm{h}$ labor & 4 & 3.9 \\
\hline \multicolumn{3}{|c|}{$\begin{array}{l}C T \text {, Computed tomography; ITA, internal thoracic artery; } C A B G \text {, coronary artery by } \\
\text { pass grafting. *Recommended staffing and equipment were " }>4 \mathrm{CV} \text { surgeons," " }> \\
\text { expert CV surgeons," " }>4 \text { cardiologists," " }>2 \text { anesthesiologists," " }>2 \text { clinical en } \\
\text { gineers," "have an intensive-care unit facility," "have a hemodialysis unit," an } \\
\text { " }>2 \text { heart-lung machines." }\end{array}$} \\
\hline
\end{tabular}

inpatient service, as well as expected outcomes for each hospital. In addition, cardiologists could use these models to determine cardiovascular surgery indications, hospital referral, and informed consent.

Effects of healthcare structures and processes in these models have further implications. Previous studies revealed the importance of teamwork in surgery as an important predictor of patient outcomes. ${ }^{17}$ Similarly, results of this study suggest that interdisciplinary professional communication may improve the outcome of cardiovascular surgery, although the best style of communication may vary by country. Because more than 2 cardiovascular surgeons routinely work together in cardiovascular surgery in Japan, ${ }^{7} 97.1 \%$ of participant hospitals have conferences with cardiovascular surgeons more than once per week. These region-specific characteristics might yield differences in the relative impact of process and structure metrics between Japan and other countries.

Although the effect of procedural volume reported by previous studies ${ }^{7,8,18}$ implied a critical role for adequate staffing and equipment, increased experience in highvolume hospitals may also be important ("practice makes perfect" hypothesis). Meeting the recommended staffing and equipment guidelines had significant effects on cardiovascular surgery outcomes (OR 0.75 in mortality model and 0.88 in composite model) independently of the hospital's annual adult cardiac surgery volume. The results of this study also suggest that improving the work environment of cardiovascular surgeons is important not only for surgeons but also for patients. Long, continuous labor is common in Japan. Although continuous labor more than 32 hours is banned by Japanese labor standards law, more than $90 \%$ of hospitals routinely impose this on surgeons. The lack of physician assistants in Japan means that surgeons have a wide range of work-related responsibilities. Thus, most surgeons in this study report having miscellaneous duties. The increased workload of doctors in Japanese hospital means that many doctors experience work burnout. A previous study indicated that hospitals operating at or over capacity may experience heightened rates of adverse events, ${ }^{19}$ and the present study also found that work

TABLE 3. Outcomes of each procedure $(N=33,821)$

\begin{tabular}{|c|c|c|c|c|c|}
\hline & $\begin{array}{c}\text { Isolated CABG } \\
\text { surgery }(\mathrm{N}=\mathbf{1 0 , 9 7 2}) \\
\end{array}$ & $\begin{array}{l}\text { Valve surgery } \\
(N=10,935)\end{array}$ & $\begin{array}{l}\text { Thoracic aortic surgery } \\
(\mathrm{N}=7559)\end{array}$ & $\begin{array}{l}\text { Other procedures } \\
\quad(N=4355) \\
\end{array}$ & $\begin{array}{c}\text { All } \\
\text { procedures } \\
\end{array}$ \\
\hline 30-d operative mortality & $2.2 \%$ & $3.7 \%$ & $8.4 \%$ & $7.1 \%$ & $4.7 \%$ \\
\hline Composite mortality or major morbidity & $14.2 \%$ & $15.5 \%$ & $31.7 \%$ & $19.5 \%$ & $19.2 \%$ \\
\hline Reoperation for any reason & $5.8 \%$ & $6.8 \%$ & $9.3 \%$ & $7.0 \%$ & $7.1 \%$ \\
\hline Stroke & $1.5 \%$ & $1.8 \%$ & $6.6 \%$ & $2.5 \%$ & $2.9 \%$ \\
\hline Renal failure newly dialysis required & $3.1 \%$ & $3.6 \%$ & $6.1 \%$ & $5.1 \%$ & $4.2 \%$ \\
\hline Deep sternal infection & $2.1 \%$ & $1.5 \%$ & $2.4 \%$ & $1.7 \%$ & $1.9 \%$ \\
\hline Prolonged ventilation & $5.8 \%$ & $6.3 \%$ & $19.2 \%$ & $9.8 \%$ & $9.5 \%$ \\
\hline
\end{tabular}

$C A B G$, Coronary artery bypass grafting. 


\begin{tabular}{|c|c|c|c|c|c|c|c|c|}
\hline & \multicolumn{4}{|c|}{ 30-d operative mortality } & \multicolumn{4}{|c|}{ Composite mortality or major morbidity } \\
\hline & \multirow[b]{2}{*}{$\boldsymbol{P}$} & \multirow[b]{2}{*}{ OR } & \multicolumn{2}{|c|}{$\mathbf{9 5} \% \mathrm{CI}$} & \multirow[b]{2}{*}{$\boldsymbol{P}$} & \multirow[b]{2}{*}{ OR } & \multicolumn{2}{|c|}{$\mathbf{9 5} \% \mathrm{CI}$} \\
\hline & & & Lower & Upper & & & Lower & Upper \\
\hline \multicolumn{9}{|l|}{ Preoperative risks } \\
\hline Age, y $(16-60,61-65,66-70,71-75,76-80,81-100)$ & .000 & 1.22 & 1.17 & 1.27 & .000 & 1.11 & 1.09 & 1.13 \\
\hline BMI $31-35 \mathrm{~kg} / \mathrm{m}^{2}$ & .057 & 1.42 & 0.99 & 2.04 & .000 & 1.76 & 1.49 & 2.08 \\
\hline $\mathrm{BMI}>35 \mathrm{~kg} / \mathrm{m}^{2}$ & .054 & 2.05 & 0.99 & 4.26 & - & - & - & - \\
\hline Smoking & - & - & - & - & .003 & 1.11 & 1.04 & 1.19 \\
\hline Diabetes & - & - & - & - & .001 & 1.15 & 1.06 & 1.25 \\
\hline Renal failure & .000 & 1.92 & 1.59 & 2.32 & .000 & 2.19 & 1.95 & 2.45 \\
\hline Renal failure requiring dialysis & .000 & 4.15 & 3.23 & 5.34 & .080 & 2.53 & 2.15 & 2.98 \\
\hline Cerebrovascular disease (recent) & - & - & - & - & .010 & 1.47 & 1.10 & 1.97 \\
\hline Infectious endocarditis & .004 & 1.64 & 1.17 & 2.30 & .006 & 1.36 & 1.09 & 1.69 \\
\hline COPD (moderate, severe) & .000 & 2.49 & 1.87 & 3.30 & .000 & 1.80 & 1.47 & 2.19 \\
\hline Extracardiac arteriopathy (peripheral) & .000 & 1.61 & 1.35 & 1.92 & .000 & 1.43 & 1.29 & 1.58 \\
\hline Extracardiac arteriopathy (thoracic) & - & - & - & - & .000 & 1.28 & 1.14 & 1.43 \\
\hline Neurologic disorder & .022 & 1.33 & 1.04 & 1.69 & .002 & 1.29 & 1.10 & 1.51 \\
\hline Congestive heart failure & .000 & 1.59 & 1.35 & 1.87 & .000 & 1.27 & 1.17 & 1.39 \\
\hline Cardiogenic shock & .000 & 1.68 & 1.35 & 2.10 & .000 & 1.60 & 1.37 & 1.88 \\
\hline NYHA IV & .000 & 1.51 & 1.24 & 1.84 & .000 & 1.74 & 1.54 & 1.96 \\
\hline Preoperative inotropic agents & .000 & 1.50 & 1.22 & 1.85 & .000 & 1.34 & 1.16 & 1.55 \\
\hline Triple vessel disease & - & - & - & - & .003 & 1.18 & 1.06 & 1.32 \\
\hline Poor LV function & .000 & 2.34 & 1.90 & 2.87 & .000 & 1.66 & 1.44 & 1.90 \\
\hline Tricuspid stenosis & .030 & 2.63 & 1.10 & 6.31 & .000 & 2.87 & 2.55 & 3.22 \\
\hline Reoperation & .000 & 2.73 & 2.26 & 3.30 & - & - & - & - \\
\hline Status (urgent) & .000 & 1.72 & 1.39 & 2.13 & .000 & 1.41 & 1.25 & 1.59 \\
\hline Status (emergency) & .000 & 2.98 & 2.46 & 3.62 & .000 & 2.06 & 1.81 & 2.33 \\
\hline Surgery type "isolated CABG" (reference) & .000 & & & & .000 & & & \\
\hline CABG combined surgery & .000 & 1.65 & 1.38 & 1.98 & .000 & 1.57 & 1.40 & 1.76 \\
\hline Surgery type "valve" & .000 & 1.60 & 1.29 & 1.99 & .001 & 1.26 & 1.10 & 1.43 \\
\hline Aortic valve + mitral valve & .001 & 2.61 & 1.96 & 3.48 & .000 & 1.85 & 1.58 & 2.18 \\
\hline Surgery type "thoracic aorta" & .000 & 2.60 & 2.05 & 3.30 & .000 & 1.61 & 1.35 & 1.92 \\
\hline Thoracic aortic surgery indication (rupture) & .000 & 4.23 & 3.23 & 5.54 & .000 & 2.58 & 2.08 & 3.19 \\
\hline Range of replacement (root) & - & - & - & - & .082 & 1.93 & 1.57 & 2.36 \\
\hline Range of replacement (arch) & .004 & 3.61 & 2.89 & 4.51 & .000 & 2.52 & 2.22 & 2.87 \\
\hline Range of replacement (distal) & .001 & 4.02 & 3.07 & 5.25 & .004 & 2.04 & 1.74 & 2.40 \\
\hline Range of replacement (descending) & .000 & 4.47 & 3.44 & 5.80 & - & - & - & - \\
\hline Range of replacement (thoracoabdominal) & .000 & 6.66 & 4.69 & 9.45 & .000 & 3.34 & 2.66 & 4.20 \\
\hline Surgery type "other procedures" & .000 & 2.16 & 1.71 & 2.72 & .029 & 1.18 & 1.02 & 1.37 \\
\hline Resection/myoplasty & .046 & 4.32 & 2.18 & 8.56 & .025 & 1.99 & 1.26 & 3.13 \\
\hline Septal perforation/rupture & - & - & - & - & .000 & 2.94 & 2.16 & 4.01 \\
\hline \multicolumn{9}{|l|}{ Structure and process of care } \\
\hline Hospital annual adult cardiac surgery volume (continuous) & .000 & 0.998 & 0.997 & 0.999 & 0.000 & 0.999 & 0.998 & 0.999 \\
\hline Recommended staffing and equipment* & .000 & 0.75 & 0.64 & 0.87 & .003 & 0.88 & 0.80 & 0.95 \\
\hline Conference with cardiologists (every day) & .073 & 0.79 & 0.60 & 1.02 & - & - & - & - \\
\hline Conference with nurse $(\geq 1 /$ wk) & - & - & - & - & .003 & $\mathbf{0 . 8 9}$ & 0.82 & 0.96 \\
\hline Intensivists concerned postsurgical management & .090 & $\mathbf{0 . 8 9}$ & 0.77 & 1.02 & .007 & 0.91 & 0.84 & 0.97 \\
\hline Have some protocols regarding drug dilution method & - & - & - & - & .000 & $\mathbf{0 . 8 3}$ & 0.77 & 0.90 \\
\hline Public hospitals & .003 & 0.80 & 0.70 & 0.93 & - & - & - & - \\
\hline Surgeons do not have miscellaneous duties & .003 & 0.80 & 0.70 & 0.93 & .095 & 0.94 & 0.87 & 1.01 \\
\hline Surgeons do not have $>32$-h labor & .032 & 0.55 & 0.32 & 0.95 & - & - & - & - \\
\hline
\end{tabular}


TABLE 5. Performance of each prediction model $(\mathrm{N}=6630)$

\begin{tabular}{lcc}
\hline & $\begin{array}{c}\text { 30-d operative } \\
\text { mortality }\end{array}$ & $\begin{array}{c}\text { Composite mortality or } \\
\text { major morbidity }\end{array}$ \\
\hline C-statistics & 0.847 & 0.744 \\
Hosmer-Lemeshow test & .790 & .485 \\
$\quad(P$ value $)$ & & \\
\hline
\end{tabular}

environment improvements might result in benefits for surgery outcomes and surgeons' quality of life.

\section{Study Limitations}

This study has several limitations. Although we chose healthcare structures and processes of care based on interviews and previous studies, specific items we chose to represent the structures and processes may not cover all relevant factors. For that reason, the validity of process and structure metrics should be reevaluated. In addition, process indicators were collected at the hospital level in this study. To obtain a better understanding of process metrics relative to standardized clinical characteristics, future studies should confirm process metrics at the patient level. Because participation in the JACVSD is voluntary, the database does not cover all cardiovascular programs in Japan. ${ }^{13}$ As such, the generalizability of our findings is limited. In addition, although predicted patient outcomes based on hospital process compliance rates may be useful for choosing a hospital, patients do not always have sufficient granularity information, such as whether the appropriate processes are in place or whether the surgical skill for a particular procedure they require is available. Patients should be cautious in their search, recognizing that individual circumstances may dictate different requirements.

\section{CONCLUSIONS}

To better understand the volume-outcome relationship in cardiovascular surgery, we focused on practices that involve cardiovascular procedures, including hospital processes and structure, which embody potential risks of perioperative mortality. Our models yielded good discrimination and calibration, so they may prove useful for hospital selection based on individual patient risks and circumstances. As for structure of care in Japan, improved surgeon work environments were also shown to be important for both surgeons and patients.
The authors thank all data managers and hospitals that participated in the JACVSD project for their efforts in data entry.

\section{References}

1. Rankin JS, Hammill BG, Ferguson TB, Glower DD, O'Brien SM, DeLong ER, et al. Determinants of operative mortality in valvular heart surgery. J Thorac Cardiovasc Surg. 2006;131:547-57.

2. Shroyer LW, Plomondon ME, Grover FL, Edwards FH. The 1996 coronary artery bypass risk model: the Society of Thoracic Surgeons Adult Cardiac National Database. Ann Thorac Surg. 1999;67:1205-8.

3. Shroyer LW, Coombs LP, Peterson ED, Eiken MC, Delong ER, Chen AY, et al. The Society of Thoracic Surgeons: 30-day operative mortality and morbidity risk models. Ann Thorac Surg. 2003;75:1856-64.

4. Edwards FH, Peterson ED, Coombs LP, Delong ER, Jamieson WE, Shroyer LW, et al. Prediction of operative mortality after valve replacement surgery. J Am Coll Cardiol. 2001;37:885-92.

5. Savage EB, Ferguson TB, Disesa VJ. Use of mitral valve repair: analysis of contemporary United States experience reported to the Society of Thoracic Surgeons National Cardiac Database. Ann Thorac Surg. 2003;75:820-5.

6. Roques F, Nashef SA, Michel P, Gauducheau E, de Vincentiis C, Baudet E, et al. Risk factors and outcome in European cardiac surgery: analysis of the EuroSCORE multinational database of 19030 patients. Eur J Cardiothorac Surg. 1999;15:816-23.

7. Miyata H, Motomura N, Ueda Y, Matsuda H, Takamoto S. Effect of procedural volume on outcome of CABG surgery in Japan. J Thorac Cardiovasc Surg. 2008; 135:1306-12.

8. Peterson ED, Coombs LP, DeLong ER, Haan CK, Ferguson TB. Procedural volume as a marker of quality for CABG surgery. JAMA. 2004;291:195-201.

9. Halm EA, Lee C, Chassin MR. Is volume related to outcome in health care? A systematic review and methodologic critique of the literature. Ann Intern Med. 2002;137:511-20.

10. Hewitt M. Interpreting the volume-outcome relationship in the context of health care quality: workshop summary Institute of Medicine. Washington DC: National Academy Press; 2000.

11. Miyata H, Motomura N, Kondo J, Takamoto S, Hasegawa T. Improving quality of healthcare in Japan: a systematic review of procedural volume and outcome literature. Biosci Trends. 2007;1:81-9.

12. Birkmeyer JD, Sun Y, Goldfaden A, Birkmeyer NJ, Stukel TA. Volume and process of care in high-risk cancer surgery. Cancer. 2006;106:2476-81.

13. Ueda Y, Fujii Y, Udagawa H. Thoracic and cardiovascular surgery in Japan during 2006. Gen Thorac Cardiovasc Surg. 2008;56:365-88.

14. Grover FL, Shroyer AL, Edwards FH, Grover FL, Shroyer ALW, Edwards FH, et al. Data quality review program: The Society of Thoracic Surgeons Adult Cardiac National Database. Ann Thorac Surg. 1996;26:1229-31.

15. Shroyer AL, Edwards FH, Grover FL. Updates to the data quality review program: The Society of Thoracic Surgeons Adult Cardiac National Database. Ann Thorac Surg. 1998;65:1494-7.

16. Hosmer DW, Lemeshow S. Assessing the fit of the model. In: Applied logistic regression. New York: Wiley \& Sons; 1989. 82-132.

17. Vincent C, Moorthy K, Sarker SK, Chang A, Darzi AW. Systems approaches to surgical quality and safety: from concept to measurement. Ann Surg. 2004;239: 475-82.

18. Miyata H, Motomura N, Ueda Y, Tsukihara H, Tabayashi K, Takamoto S. Toward quality improvement of thoracic aortic surgery: estimating volumeoutcome effect from nationwide survey. Eur J Cardiothorac Surg. 2009;36: 517-21.

19. Weissman JS, Rothschild JM, Bendavid E, Sprivulis P, Cook EF, Evans RS, et al. Hospital workload and adverse events. Med Care. 2007;45:448-55. 